\title{
Uma Game Engine para Aventuras Pedagógicas Locativas em Realidade Aumentada
}

\author{
Otávio Lube dos Santos, Jadson do Prado Rafalski, Crediné Silva de Menezes \\ Programa de Pós-Graduação em Informática - Universidade Federal do Espírito \\ Santo (UFES) \\ Av. Fernando Ferrari, 514, Goiabeiras - Vitória - ES - CEP 29075-910 \\ \{olube,jrafalski \}@inf.ufes.br, credine@gmail.com
}

\begin{abstract}
The increasing use of mobile devices and their sensors, such as GPS, in addition to the connectivity of these devices to the Internet, encouraged the development of games for mobile platforms. An essential component for running games is known as engine, or simply, the execution environment of the game, by which rules, user interactions, and all the mechanics are processed and displayed on the screen of the device with the aid of graphics libraries. This paper presents a game engine for performing locative games with augmented reality, component of ARLA, an environment for creating and live locative adventures with augmented reality, developed in the context of a project of the same name, built with the purpose of promoting the construction of pedagogical architectures.
\end{abstract}

Resumo. O crescente uso de dispositivos móveis e seus sensores, como o GPS, além da conectividade destes equipamentos com a Internet, incentivou o desenvolvimento de jogos para plataformas móveis. Um componente essencial para a execução de jogos é conhecido como engine, ou simplesmente, ambiente de execução do jogo, por meio do qual as regras, interações de usuários e toda a mecânica são processadas e exibidas na tela do dispositivo com auxílio de bibliotecas gráficas. Este trabalho apresenta uma game engine para realização de jogos locativos com realidade aumentada, componente do ALRA, um ambiente para criação e realização de aventuras locativas com realidade aumentada, desenvolvido no contexto de um Projeto de mesmo nome, construído com o objetivo de favorecer a construção de arquiteturas pedagógicas.

\section{Introdução}

Os sistemas computacionais e a Internet transformaram significativamente a sociedade contemporânea. A forma como são utilizados somada ao constante avanço tecnológico proporcionam uma atualização paradigmática de como as pessoas se organizam com uso da tecnologia. Hoje, a leitura diária das notícias, a organização de uma agenda pessoal, uma partida de xadrez e muitas outras atividades são realizadas por meio de computadores e dispositivos móveis conectados via Internet, em grande parte das vezes de forma colaborativa. Aplicações como Waze [Waze, 2013] e Runkeeper [Runkeeper, 2013] são exemplos de sistemas colaborativos para compartilhamento de informações do trânsito e de treinos esportivos, respectivamente. 
Alguns sistemas chamam a atenção dada a imersão com a qual seus usuários interagem entre si e com o próprio ambiente do sistema. Nestes Mundos Virtuais os usuários são representados por seus avatares ${ }^{1}$.

Segundo Thomaz et. al., 2005,

[...] Constatou-se que a realidade virtual na WEB, pode além de simular o real, proporcionar experiências inusitadas, incentivar a colaboração entre os sujeitos sem impedimentos geográficos e propiciar a constituição de narrativas avaliativas dos próprios percursos de aprendizagem.

A utilização de sistemas com tais características se acentuou ainda mais com o advento da computação ubíqua, pela qual os usuários, por meio de dispositivos móveis interconectados à Internet sem fio, permeiam diariamente seus mundos virtuais. Alguns destes mundos constituem jogos com variados propósitos, desde os meramente dedicados ao entretenimento até aqueles com fins explicitamente educacionais [Santos, 2013].

Buscando contribuir com esse tipo de inserção desta "tendência" no contexto educacional concebemos o Projeto ALRA, que tem por finalidade oferecer um ambiente para desenvolvimento e realização de aventuras locativas com realidade aumentada no contexto educacional.

Neste artigo apresentamos um dos componentes essenciais do ambiente para realização de jogos, a sua Game Engine, elemento responsável pela implementação da mecânica de um jogo. O texto está organizado da seguinte forma: o capítulo um traz este texto introdutório. No capítulo dois fazemos uma reflexão sobre jogos e aprendizagem. No capítulo três apresentamos a definição de realidade aumentada. $\mathrm{O}$ ALRA é apresentado no capítulo quatro. O capítulo cinco traz exemplos de aventuras que podem ser criadas no ambiente. $\mathrm{O}$ capítulo seis traz as conclusões do trabalho.

\section{Jogos e Aprendizagem}

Inserir novas tecnologias na educação tem sido uma prática comum com o crescente desenvolvimento tecnológico. Todavia, é sabido que a mera utilização de tecnologia dentro da escola, sem a devida associação a pedagogias elaboradas para aquelas práticas não proporciona uma vantagem na aprendizagem dos estudantes [Santos et. al., 2011, apud Valente, 1993].

A motivação é uma condição necessária para a aprendizagem. Toda pessoa engajada num objetivo se torna fascinada em alcançá-lo, o que faz de jogos um excelente instrumento para aprender, diferentemente da escola tradicional [Prensky, 2003]. Jogos permitem que os estudantes sejam produtores de seu próprio conhecimento e não apenas consumidores de informação [Gee, 2003]. Podem desenvolver habilidades cognitivas dos jogadores de forma que estes possam transferir o conhecimento do mundo virtual para o mundo real [Gee, 2007].

Jogos são, portanto, uma excelente forma de construir conhecimento. Proporcionam desafios em mundos regidos por regras compreensíveis que vão sendo entendidas no decorrer do jogo. Koster, 2005, os define como:

\footnotetext{
${ }^{1}$ Representações gráficas de um sujeito em um mundo virtual por meio da qual este interage e se faz parte integrante do mundo.
} 
[...] quebra-cabeças para resolver, assim como tudo mais que encontramos na vida. Eles estão na mesma ordem que aprender a dirigir um carro ou aprender as tabuadas. Aprendemos os padrões subjacentes, desvendamos totalmente, e os arquivamos, para que possam ser novamente usados, se necessário. A única diferença real entre os jogos e a realidade é que os riscos são menores com jogos [Koster, 2005, tradução nossa].

Apoiados em estratégias pedagógicas adequadas os jogos podem, definitivamente, constituir uma boa ferramenta para apoiar a construção de conhecimento. Podemos citar como estratégias as Arquiteturas Pedagógicas (APs), nas quais a ideia central é a modelagem de situações para promover a aprendizagem por meio da união de abordagem pedagógica, software, internet, inteligência artificial, educação a distância e, principalmente, didáticas flexíveis, maleáveis, adaptáveis e diferentes enfoques temáticos [Carvalho, Nevado, Menezes, 2005].

\section{Jogos Locativos em Realidade Aumentada}

Nos últimos anos houve uma explosão no número de novos jogos facilitados por dispositivos móveis baseados na posição do usuário rastreada por meio de sensores de satélites GPS [Avouris, Yiannoutsou, 2012].

Kiefer, Matyas e Schlieder (2006) definem jogo locativo como:

Um jogo baseado na posição (jogo locativo) é um jogo no qual há um suporte a uma tecnologia de posicionamento e que integra a posição de (um ou vários) jogadores como elementos do jogo principal e suas regras [tradução nossa].

Location Based Games (LBG), como também são chamados, são fortemente ligados ao contexto no qual se situam, de forma que a posição se junta à realidade específica do enredo em que se encontra, proporcionando uma forte sensação de imersão no jogo realizado. Esta presencialidade nesta modalidade de jogo é reforçada pelo conceito de Realidade Aumentada (RA).

RA é a fusão de recursos gráficos de computador, visuais e multimídia, que realçam a percepção do usuário sobre o mundo real, adicionando informações do mundo virtual [Kirner, Kirner, 2008]. É a inserção de objetos virtuais no mundo real por meio de um dispositivo computacional, de forma que a interface do usuário torna-se aquela utilizada no mundo real, adaptada para visualizar e manipular os objetos virtuais colocados no seu espaço [Thomaz et. al., 2005].

\section{O Projeto ALRA}

$\mathrm{Na}$ literatura há vários ambientes para a construção e experimentação mundos virtuais e jogos. Dentre os principais, citamos o Second Life [Second Life, 2013] representando ambientes 3D, o Klik\&Play [Lionet, Lamoureux, 1994] como ambiente 2D e o Aris [Aris Games, 2013] como ambiente baseado na localização. Entretanto estes ambientes possuem suas restrições, relacionadas a dificuldade de criação de edição de enredos interessantes, a falta de conectividade entre os usuários do jogo e a impossibilidade de acompanhamento dos jogadores e reutilização dos componentes do jogo, respectivamente. A partir destas observações de sistemas relacionados, identificou-se a oportunidade de construir um novo ambiente que contribuísse para atenuação dos 
problemas dos citados e que, além disso, fosse adaptável a modificações futuras como um framework aberto.

O ALRA é uma vertente de um projeto mais amplo de construção e execução de jogos em várias plataformas denominado Clic\&Ação [Silva et. al., 2012]. Nesta vertente, como já mencionado, as aventuras são locativas com uso de realidade aumentada, de forma que os aventureiros interagem com os objetos do ambiente que são dispostos num mapa em seu dispositivo móvel. As premissas básicas do ambiente são:

1. As aventuras serão locativas com base num mapa simulado no dispositivo móvel do usuário. Nele serão dispostos os objetos e ferramentas com os quais os aventureiros deverão interagir para cumprir suas missões e conquistar seus resultados;

2. O avatar do aventureiro é mostrado na posição no mapa indicada pelo GPS do dispositivo móvel;

3. As interações entre os aventureiros e o mundo virtual em questão são persistentes e visíveis a todos os aventureiros daquela aventura, ou seja, é um ambiente multiplayer ou multiaventureiro;

4. Os aventureiros, além de vivenciar aventuras, podem criá-las por meio de um ambiente web colaborativo;

5. Os aventureiros vivenciam uma aventura com auxílio de seus dispositivos móveis e um executor de aventuras, descrito neste artigo.

A arquitetura do ALRA pode ser observada abaixo, composta por dois componentes principais: o ambiente web de criação de aventuras e o executor destas aventuras, desenvolvido inicialmente para dispositivos que suportam o sistema operacional Android [Android Developers, 2013].

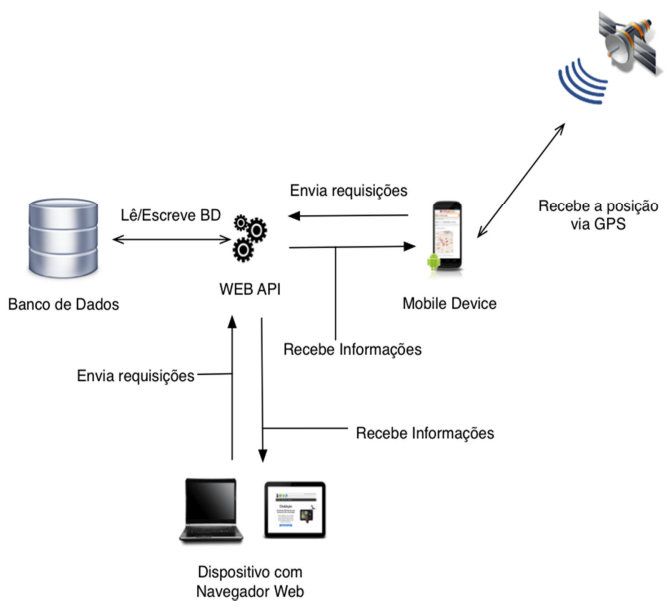

Figura 1 - Arquitetura do ALRA [Santos, 2013]. 
Assim, aventuras podem ser criadas num ambiente web e executadas num dispositivo móvel, segundo um modelo de dados compartilhado para as duas aplicações. Nele são definidos os papeis dos usuários na aventura e todas as suas contribuições realizadas, seja como criadores, editores ou aventureiros de uma aventura realizada. Neste artigo descreveremos mais a fundo o executor de aventuras pedagógicas do ALRA.

\subsection{O Executor de Aventuras do ALRA}

$\mathrm{O}$ executor de aventuras do ALRA consiste na engine que descreve como as aventuras serão lidas e avaliadas do banco de dados para o dispositivo móvel. Game Engines geralmente são um conjunto de ferramentas e componentes de execução em tempo real que promovem o funcionamento de um jogo. Por serem realizadas em um dispositivo móvel, a integração do executor de aventuras com o banco de dados da aplicação ocorrem por intermédio de uma API WEB desenvolvida na linguagem PHP. Por meio de requisições HTTP o executor, desenvolvido inicialmente para a plataforma Android, recebe as informações no formato JSON, as decodifica e insere num banco de dados aulixiar local e executa em tempo real a aventura junto ao aventureiro. A arquitetura do executor pode ser observada abaixo. O mapa no qual ocorrem as aventuras foi construído com auxílio da Google Maps API [Android Developers, 2013], cujas funções pré-definidas serviram como framework para a elaboração das interações com o mapa.

\begin{tabular}{|c|c|c|c|c|c|c|c|}
\hline \multicolumn{8}{|c|}{ Subsistemas Especificos das Aventuras } \\
\hline Niveis & Armadilhas & Missöes & Veículos & Avatares & Persona & agens & Poderes \\
\hline \multicolumn{8}{|c|}{ Recursos da Aplicacäo } \\
\hline Telas do & Sistema & Mapas & Editores & Grupo & Missô & ةes & Requisitos \\
\hline \multicolumn{8}{|c|}{ Gerenciador de Recurso da Plataforma Móvel } \\
\hline \multicolumn{8}{|c|}{ Bibliotecas Principais } \\
\hline SQLite & JSON Parser & \multicolumn{2}{|c|}{ HTTPRequest } & Gráficos & \multicolumn{3}{|c|}{ Avaliacão Lógica de Requisitos } \\
\hline \multicolumn{8}{|c|}{ Camada da Plataforma Móvel } \\
\hline \multicolumn{2}{|c|}{ Alocacao de Memoria } & \multicolumn{2}{|c|}{ Funcoes Matematicas } & \multicolumn{2}{|c|}{ Operacoes de $1 / 0$} & \multicolumn{2}{|c|}{ Comunicacão TCP } \\
\hline \multicolumn{2}{|c|}{ Sistema de Arquivos } & \multicolumn{2}{|c|}{ Tipos de Dados } & sers (XML, & JSON) & \multicolumn{2}{|c|}{ Gerenciador de Mídias } \\
\hline \multicolumn{8}{|c|}{ SDK de Terceiros (Android, iOS Development Kit, Google Maps API) } \\
\hline \multicolumn{8}{|c|}{ Sistema Operacional (Android, ios, Windows, etc...) } \\
\hline \multicolumn{8}{|c|}{ Dispositivos Multimídia e Sensores (Camera, Microfone, GPS, Giroscópio, Acelerômetro, etc.) } \\
\hline \multicolumn{8}{|c|}{ Hardware (Dispositivo Móvel) } \\
\hline
\end{tabular}

Figura 2 - Arquitetura da Engine do ALRA [Santos, 2013].

A figura 2 mostrada acima mostra a arquitetura do ambiente de execução em tempo real do ALRA, estruturada em camadas. As camadas inferiores constituem elementos de hardware software relativos ao dispositivo móvel em questão. A Camada da Plataforma móvel possui elementos construídos para interagir com a camada imediatamente abaixo. As Bibliotecas Principais são bibliotecas programadas para 
prover as funcionalidades da engine. Os Recursos da Aplicação constituem às possíveis funcionalidades do sistema enquanto os Subsistemas Específicos das Aventuras mostram os elementos interpretados pela engine no ALRA.

O fluxo de execução de uma aventura pode ser observado na figura abaixo:

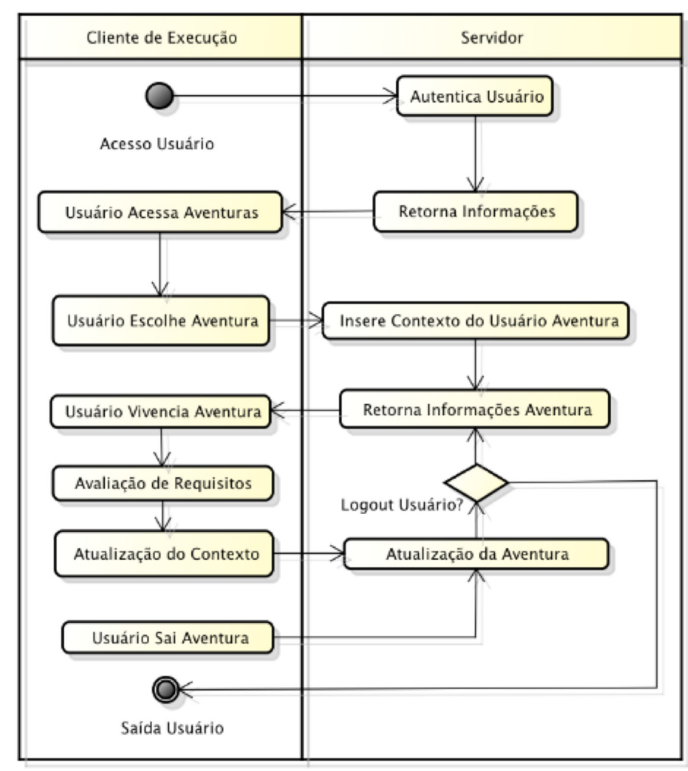

Figura 3 - Fluxo de execução de uma aventura no ALRA [Santos, 2013].

$\mathrm{O}$ executor interage com os sensores e funcionalidades disponíveis no dispositivo móvel, como GPS, giroscópio, câmera, microfone, alto-falantes, etc. Um aventureiro pode realizar aventuras públicas ou para as quais foi designado, definidas pelo ambiente web de criação de aventuras. Nessas aventuras são estabelecidas missões, que podem ser cumpridas em grupos pré-definidos ou não. A realização destas missões está atrelada ao cumprimento ou não de requisitos associados a elas, de forma que o executor avalie logicamente algumas condições. Um exemplo disto é a necessidade de estar uma determinada quantidade de metros próximo a um objeto para interagir com ele. Objetos ou ferramentas específicas podem ser necessários para o cumprimento de requisitos ou missões. Estes podem ser obtidos durante a aventura, ora disponível no próprio mapa, ora adquiridas na realização de outras missões.

A realização de missões também pode fazer com que o aventureiro mude de nível, abrindo um novo leque de missões que podem ser cumpridas. Durante todo o processo os aventureiros possuem ferramentas para a produção individual, como um diário para anotações pessoais, sua câmera e microfone para aquisição de mídias com auxílio do dispositivo móvel. Estas produções individuais foram denominadas no contexto do ALRA de Unidades de Produção Individual (UPIs). Elas ainda podem ser compartilhadas entre os aventureiros que participam de uma determinada aventura simultaneamente, por meio do mapa.

\section{Uma Aventura para Recepção de Calouros no ALRA}

Construímos o ALRA com o propósito de oferecer aos professores (e seus alunos) a possibilidade de conceber e vivenciar novas experiências de aprendizagem, que unam o uso de equipamentos moveis e realidade ampliada, através de uma Arquitetura 
Pedagógica que temos denominado de Aventuras Pedagógicas. Chamamos de aventura por ser um jogo no qual se cumpre missões viajando entre espaços diversos com regras pré-determinadas onde se utiliza ferramentas previamente dadas ou mesmo obtidas durante a aventura. Os objetos e prêmios alcançados podem ser guardados para serem aproveitados em outras aventuras que o jogador, ou como chamamos no ambiente, aventureiro, por ventura realizar.

A aventura de recepção dos calouros numa universidade tem como objetivo prover uma forma inovadora de recepcionar os novos estudantes à universidade por meio de seus dispositivos móveis. Consiste em uma aventura pública disponível no ambiente ALRA para que qualquer pessoa possa conhecer melhor a uma universidade e suas características.

Além de vários objetos indicadores da história da universidade e com informações relevantes sobre o funcionamento da mesma, a aventura possui missões para os calouros, que vão desde encontrar o departamento para realização da matrícula até a inscrição na biblioteca central e acesso a links com apresentação dos professores em vídeo com ajuda de QR Codes ${ }^{2}$.

A medida que o calouro progride em suas missões, este evolui de nível, fazendo com que novas missões apareçam para serem realizadas. O cumprimento das missões é recompensado com informações importantes, prêmios e acúmulo de pontos que culmina na evolução do nível. Em sua jornada os aventureiros ainda podem encontrar armadilhas, como é o caso do trote. Em determinado nível de experiência o calouro é chamado para um determinado lugar onde leva um trote virtual, no qual perde todos os seus pontos, todavia são conduzidos ao próximo nível da aventura, no qual novos desafios podem ser programados no ambiente de autoria do ALRA.

Durante a aventura é possível gravar sons e vídeos, tirar fotos e escrever anotações. Estas UPIs podem ser jogadas no mapa para que qualquer aventureiro visualize. Também é possível visualizar todos os aventureiros online na aventura naquele momento, representados por seus avatares selecionados nos perfis descritos no ambiente de autoria.

Os aventureiros ainda podem guardar seus objetos e produções numa mochila virtual. Estes objetos podem ser reutilizados em outras aventuras caso o criador da aventura permita sua reutilização no ambiente de autoria.

\footnotetext{
${ }^{2}$ QR Code é um código de barras em duas dimensões que pode ser escaneado na maioria dos aparelhos ceulalares com câmera fotográfica. Após sua decodificação, é transformado num texto ou num hyperlink [QR CODE, 2013].
} 


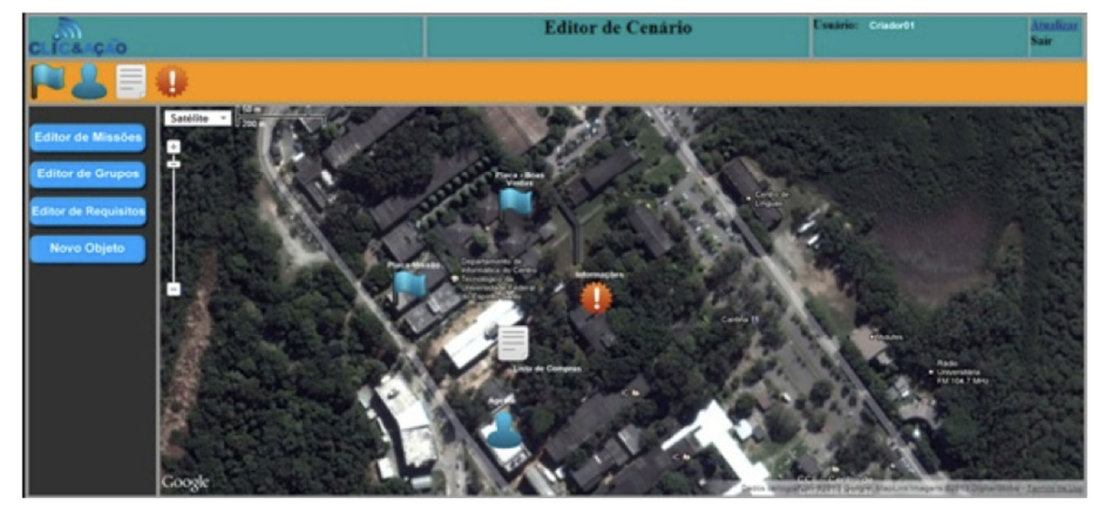

Figura 4 - Composição da Aventura na universidade no ambiente de autoria do ALRA [Rafalski, 2013].

\section{Conclusões}

O ALRA encontra-se desenvolvido e disponível para download em celulares que utilizam Android através do aplicativo Google Play [Google Play, 2013]. Pode ser desenvolvido para outros sistemas operacionais móveis seguindo-se a documentação disponível na página do projeto [Google Code, 2013]. Possui código aberto de maneira que outras pessoas possam adaptá-lo às suas necessidades.

Na Figura 5 é possível verificar a configuração inicial de aventura e dois participantes a realizando no ALRA. O ambiente se atualiza conforme o deslocamento geográfico dos aventureiros e as interações com o sistema realizadas através do dispositivo móvel.

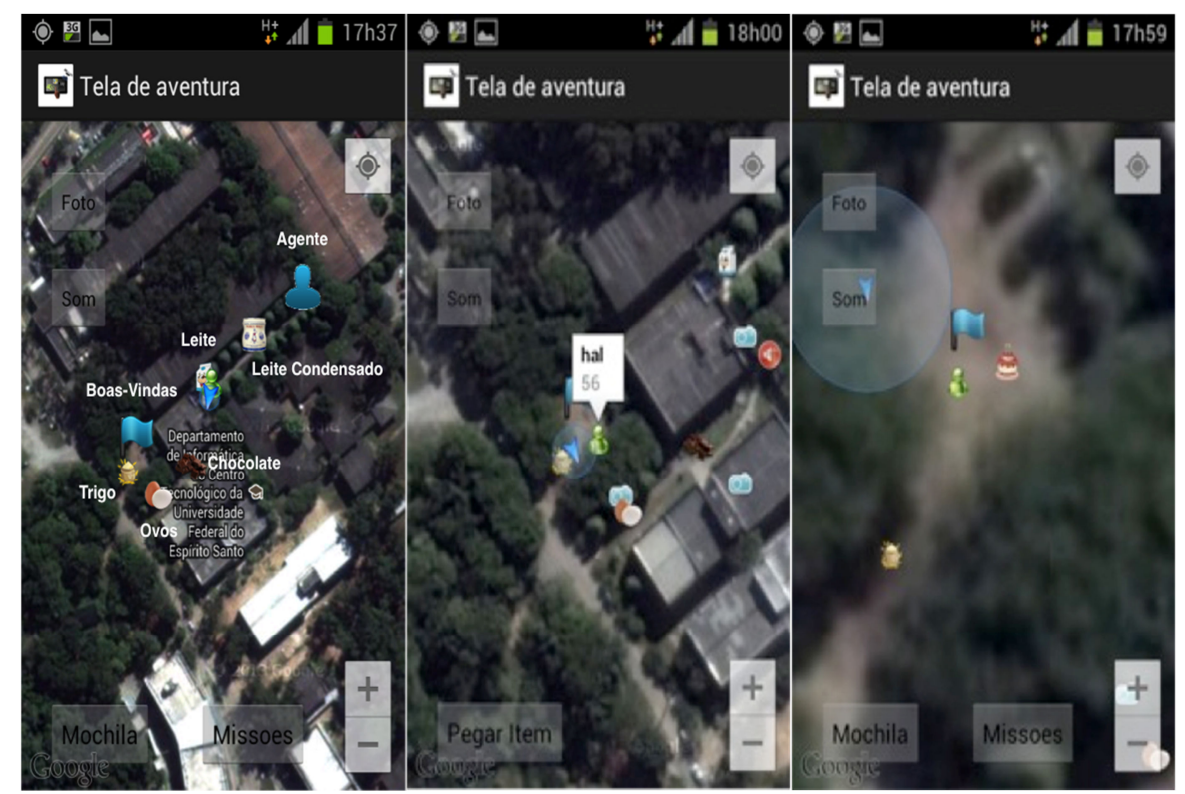

Figura 5 - Aventura sendo realizada no ALRA [Santos, 2013].

Os resultados encontrados na utilização do sistema mostraram que a imersão dos aventureiros está relacionada à complexidade da aventura criada. A modelagem integrada do ambiente de autoria e do ambiente de execução (o qual fora aqui apresentado) possibilita que aventuras complexas como a da recepção de calouros na 
universidade sejam modeladas de forma a garantir o engajamento dos estudantes na sua realização.

A possibilidade de interagir com outros participantes nas aventuras possibilita também um ambiente de cooperação dos aventureiros, os quais podem realizar missões em grupo distribuindo inclusive os requisitos destas missões. O ALRA pode ser então utilizado como suporte à Arquiteturas Pedagógicas baseadas em jogos locativos, uma vez que proporciona um ambiente de autoria no qual os estudantes podem também criar suas próprias aventuras e divulgá-las. $\mathrm{O}$ ambiente de execução permite a reutilização de itens e ferramentas entre aventuras, desde que sejam programadas para tal. Os aventureiros podem também colecionar suas produções multimidiáticas e compartilhálas em suas aventuras.

Apesar do enfoque educacional dado ao ALRA, este ambiente pode ser utilizado para a elaboração de aplicações em qualquer outra área da atividade humana. Para o turismo, por exemplo, podemos criar roteiros interessantes nos quais os aventureiros colecionam UPIs e se localizam baseados em sua posição e nos elementos colocados no mapa. Agentes programados podem auxiliar no roteiro, dando instruções aos aventureiros, tornando a viagem mais interessante e interativa. Além disto, o guia turístico pode acompanhar os turistas a partir de um tablet e uma tela de acompanhamento web.

Novos módulos podem ser programados para agregar funcionalidades ao ambiente. Algumas já foram previstas e são compartilhadas na seção seguinte. Outras podem surgir de acordo com a necessidade e a criatividade dos próprios aventureiros em suas experiências no sistema.

\section{Referências}

Android Developers. Plataforma de desenvolvimento Android. Disponível em: < http://developer.android.com/>. Acessado em: 01/08/2013.

Aris Game. Site oficial. Disponível em <http://arisgames.org/>. Acesso em: 01/08/2013.

Avouris, N.; Yiannoutsou, N.. A review of Mobile Location-Based Games for Learning across Physical and Virtual Spaces. Journal of Universal Computer Sciense, vol. 18, no. 15, pp. 2120-2142, 2012.

Gee, J. P. What Video Games Have to Teach Us About Learning and Literacy. ACM Computers in Entertainment, Vol. 1, No. 1, BOOK 1, 2003.

Gee, J. P.. Good Video Games + Good Learning. Peter Lang. New York, 2003.

Google Play. Serviço de distribuição de software da Google. Disponível em < https://play.google.com>. Acessado em: 01/08/2013.

Google Code. Página do projeto Clic\&Ação. Disponível em: $<$ https://code.google.com/p/clicacao-project/>. Acessado em 01/08/2013.

Kiefer, P.; Matyas, S.; Schlieder C.. Systematically exploring the design space of location-based games. In Pervasive Workshop Proceedings, Poster presented at PerGames2006, pages 183-190, Dublin, Ireland, May 2006. 
Kirner, C.; Kirner, T. G.. Virtual Reality and Augmented Reality Applied to Simulation Visualization. In: El Sheikh, A.A.R.; Al Ajeeli, A.; Abu-Taieh, E.M.O.. (Ed.). Simulation and Modeling: Current Technologies and Applications. 1 ed. HersheyNY: IGI Publishing, 2008, v. 1, p. 391-419.

Koster, R. A Theory of Fun for Game Design. Ed. Paraglyph Press. ISBN: 1-932111-972. 2005 .

Lionet, F.; Lamoureux, Y. Klik and Play. Maxis. W3C. World Wide Web Consortium, 1994. Disponível em <http://www.w3.org/>. Acesso em 19/08/2012.

Nevado, R.A., Dalpiaz, M., Menezes, C.S. (2009). Arquiteturas Pedagógicas para Construção Colaborativa de Conceituações, XXIX Congresso da Sociedade Brasileira de Computação, Bento Gonçalves - RS.

Prensky, M. (2003). Digital game-based learning. Computers in Entertainment. Editora: McGraw-Hill. University of Califórnia.

QR Code. QR Code Standardization. Disponível em < http://www.qrcode.com/en/qrstandard.html>. Acessado em 02/08/2013.

Rafalski, J. P. Um Ambiente Colaborativo para Criação de Aventuras Locativas com Realidade Aumentada. Dissertação de Mestrado. PPGi/UFES. 2013.

Runkeeper. The Personal Trainer in Your Pocket. Disponível em: $<\mathrm{http}$ ://www.runkeeper.com/>. Último acesso em 01/09/2013.

Santos, O. L.; Rafalski, J. P.; Silva, H. F. A.; Cury, D.; Menezes, C. S. Mundos Virtuais para Construção de Arquiteturas Pedagógicas. Workshop WAPSEDI Anais do XXII SBIE - XVII WIE, 2011.

Santos, O. L. Um Ambiente para Realização de Aventuras Locativas com Realidade Aumentada. Dissertação de Mestrado. PPGi/UFES. 2013.

Second Life. Site Oficial do Second Life. Disponível em: $<$ http://secondlife.com/>. Acessado em: 01/08/2013.

Silva, H. F. A., Santos, O. L.; Rafalski, J. P.; Cury, D.; Menezes, C. S. Clic\&Ação: Um Ambiente para a Construção Colaborativa de Micromundo. Anais do 23 SBIE, 2012.

Thomaz, A. R.; Maraschin, C.; Zaniol, E.; Schuch, E. M. M.. Dispositivos de imersão em ambientes de realidade virtual. Revista brasileira de informática na educação. Florianópolis. Vol. 13, n. 2, p.21-32, 2005.

Valente, J. A. (1993). Por Quê o Computador na Educação. Em J.A. Valente (Org.), Computadores e Conhecimento: repensando a educação (pp. 24-44). Campinas, SP: Gráfica da UNICAMP.

Waze. Outsmarting Traffic Together. Disponível em: <http://www.waze.com/>. Último acesso em 01/09/2013. 